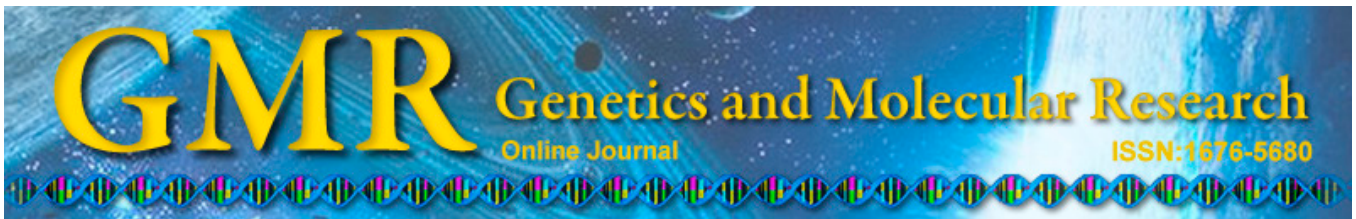

\title{
Clinical observational study of conformal radiotherapy combined with topotecan chemotherapy in patients with platinum- resistant recurrent ovarian cancer
}

\author{
P. Wei ${ }^{1}$, Z.H. Zhang' ${ }^{2}$ L. Li ${ }^{3}$, X.L. Du ${ }^{1}$, C.P. Shan ${ }^{4}$ and X.G. Sheng ${ }^{1}$ \\ ${ }^{1}$ Department of Gynecologic Oncology, Shandong Tumor Hospital and Institute, \\ Jinan, Shandong Province, China \\ ${ }^{2}$ Department of Occupational Health and Occupational Medicine Institute, \\ Jinan, Shandong Province, China \\ ${ }^{3}$ Central People's Hospital of Tengzhou City, Jining Medical College, \\ Tengzhou, Shandong Province, China \\ ${ }^{4}$ Oncology Department, Affiliated Hospital of Jining Medical College, Jining, \\ Shandong Province, China
}

Corresponding author: X.G. Sheng / Z.H. Zhang

E-mail: weiping_wp@yeah.net / zyhwp@163.com

Genet. Mol. Res. 14 (2): 3833-3842 (2015)

Received May 28, 2014

Accepted October 9, 2014

Published April 22, 2015

DOI http://dx.doi.org/10.4238/2015.April.22.12

\begin{abstract}
This retrospective study aimed to observe the curative effect and adverse reactions of three-dimensional conformal radiotherapy combined with topotecan chemotherapy in patients with platinum-resistant recurrent epithelial ovarian carcinoma. The chemoradiotherapy group $(\mathrm{N}=22)$ received $15 \mathrm{mv} \mathrm{X}$-rays with 1.8 to $2.0 \mathrm{~Gy} / \mathrm{f} / \mathrm{d}$ radiation, 5 times per week. The total dose was 45 to $65 \mathrm{~Gy}$; the median dose was $52.5 \mathrm{~Gy}$. Topotecan chemotherapy $\left(2.0 \mathrm{mg} / \mathrm{m}^{2}\right)$ was administered after the first week of radiotherapy
\end{abstract}


on days 1,8 , and 15 ; it was repeated every 28 days. The only chemotherapy group $(\mathrm{N}=20)$ received topotecan chemotherapy $(4.0$ $\mathrm{mg} / \mathrm{m}^{2}$ ) in the first week, and the dose was administered on days 1,8 , and 15 ; it was repeated every 28 days. The median follow-up times were 18.5 months ( 2 to 37.7 ) and 10.8 months (1.5 to 29.6) in the chemoradiotherapy and in the only chemotherapy groups, respectively. The total response rates were $42.1 \%(8 / 19)$ and $11.1 \%$ $(2 / 18)$, respectively. The clinical benefit rates were $68.4 \%(13 / 19)$ and $22.2 \%(4 / 18)$, respectively, with significant difference $(\mathrm{P}<$ $0.05)$. The median disease progression-free periods were 9.8 and 6.6 months, respectively, with significant difference $(\mathrm{P}<0.001)$. The median survival times were 19.7 and 12.5 months, respectively, with significant difference $(\mathrm{P}<0.05)$. The degrees of digestive tract reaction rates were $26.3 \%(5 / 19)$ and $16.7 \%(3 / 18)$, whereas the hematology toxicity rates were $21.1 \%(4 / 19)$ and $22.2 \%(4 / 18)$, respectively, with no significant difference $(\mathrm{P}>0.05)$. As threedimensional conformal radiotherapy combined with topotecan chemotherapy had good curative effect on platinum-resistant recurrent epithelial ovarian cancer, with mild adverse reactions, this technique can be used as a remedial measure.

Key words: Recurrent epithelial ovarian carcinoma; Platinum resistance; Three-dimensional conformal radiotherapy; Topotecan chemotherapy; Curative effect

\section{INTRODUCTION}

In ovarian cancer, especially epithelial ovarian cancer, $70 \%$ of patients reach advanced stage once the disease is detected. Despite a better response to cytoreductive surgery and combined paclitaxel- and platinum-based postoperative chemotherapy, most patients have a relapse within 2 years (Gardner and Jewell, 2011). Treatment of recurrent epithelial ovarian cancer is difficult, especially in cases of platinum-resistant pelvic recurrence and lymph node metastases. Effective treatment is lacking. Three-dimensional conformal radiotherapy (3DCRT) is a new radiotherapy technique developed in recent years. It can increase local radiation dose to the tumor and reduce the dose to normal tissues, thereby improving the therapeutic effect and reducing complications. Topotecan (TPT) is a second-line chemotherapy drug for ovarian cancer. It has no paclitaxel or platinum crossresistance and it is an effective choice for patients with recurrent paclitaxel and platinum resistance (Sehouli and Oskay-Ozcelik, 2009; Lorusso et al., 2010; Naumann and Coleman, 2011). Between June 2008 and June 2011, our hospital used 3DCRT combined with TPT chemotherapy to treat 22 cases of platinum-resistant and recurrent epithelial ovarian cancer. Twenty patients receiving single-agent TPT chemotherapy during the same period constituted the control group. The efficacy and adverse reactions of the groups are presented herein. 


\section{MATERIAL AND METHODS}

\section{Materials}

\section{Enrolled conditions}

After patients underwent cytoreductive surgery and received multiple courses of chemotherapy, pelvic retroperitoneal lymph node metastasis or recurrence were still observed. The patients specifically included were those who received two or more cycles of cisplatin + doxorubicin + cyclophosphamide (PAC), cisplatin + cyclophosphamide (PC), or paclitaxel + cisplatin (TC). Either the tumor still progressed or recurrence appeared within 6 months of chemotherapy after six to eight sessions of PAC, PC, or TC treatment. Physical examination, B-mode ultrasonography, computed tomography (CT), and magnetic resonance imaging (MRI) did not reveal any liver, lung, bone, brain, or other distant metastasis, or intestinal symptoms. The Karnofsky score was $>70$ points.

\section{Clinical data}

Between June 2008 and June 2011, 42 cases of recurrent epithelial ovarian cancer were observed in Shandong Tumor Hospital. The patients were treated with cytoreductive surgery and multiple courses of postoperative chemotherapy. They were commonly resistant to platinum-based chemotherapy. There were 21 cases of pelvic recurrence, 9 of retroperitoneal lymph node metastases, and 12 of pelvic recurrence and retroperitoneal lymph node metastasis. The tumor infiltrated the pelvis or surrounding organs, and surgical resection indications were not detected. Clinical symptoms mainly included lumbosacral pain, abdominal pain, abdominal bulge discomfort, irregular vaginal bleeding, and lower extremity edema.

\section{Methods}

\section{Chemotherapy}

In the chemoradiotherapy group, $2.0 \mathrm{mg} / \mathrm{m}^{2} \mathrm{TPT}$ chemotherapy was administered after the first week of radiotherapy. For the chemotherapy group, $4.0 \mathrm{mg} / \mathrm{m}^{2} \mathrm{TPT}$ chemotherapy was administered in the first week. Both doses were administered on days 1,8 , and 15 . The process was repeated every 28 days. If tumor progression or intolerance to toxicity appeared after two cycles of chemotherapy in patients, a change of chemotherapy was considered.

\section{Radiotherapy methods}

Placement: Patients were in a supine position with both hands on the back of the head. Vacuum-forming mats were used to the fix position, while a stereotactic frame was used to fix surface markers and reduce placement errors.

Abdominal CT Location: CT was performed from the abdomen to the lower pelvic region. The thickness was $0.5 \mathrm{~cm}$. Scanned image data were transferred via the local area network to the planning system workstations.

Target and organs at risk definition: Before making a three-dimensional plan, the tar- 
gets were outlined at each CT level. The gross target volume (GTV) was the location with recurrent tumor and lymph node metastases. Based on the GTV, the outer 0.5- to 1.0-cm margin was taken as the planning target volume (PTV). Meanwhile, the organs at risk (OAR) were identified. These were the small intestine, rectum, bladder, spinal cord, and both femoral heads.

Design and implementation of treatment plan: The CT image data were stored in ADAC Pinnacle planning system (Philips Radiation Oncology Systems, Milpitas, CA, USA) for image reconstruction. The geometric center of the PTV was taken as the field center. The Varian EX linear accelerator power dynamic multileaf collimator was used; 6 to 10 coplanar radiation fields were set, and $15 \mathrm{MV}$ X-rays were used for irradiation. A three-dimensional treatment planning system was used to calculate the dose distribution, with a $90 \%$ isodose curve surrounding $99 \%$ GTV. The field image system was used for validation. The PTV prescription dose was 45 to 65 Gy. The median dose was $53.5 \mathrm{~Gy}$, with 1.8 to 2.0 Gy fractions delivered 5 times per week.

\section{Curative effect evaluation}

At least two cycles of chemotherapy treatment were used in the chemoradiotherapy group, 1.5 to 2 months after 3DCRT. Clinical examination, ultrasonography, or CT was used for evaluation in this group. The overall survival rate was the period from receiving chemoradiotherapy or chemotherapy alone to death. The progression-free survival time was the period from receiving chemoradiotherapy or chemotherapy alone to disease progression. Evaluation criteria were as follows: complete response (CR), where the tumor disappeared completely for at least 4 weeks, and no new lesion was found; partial response (PR), where tumor regression was more than $50 \%$ for at least 4 weeks, and no new lesion was found; stable disease (SD), where there was less than $50 \%$ tumor regression or less than $25 \%$ of tumor growth; and progressive disease (PD), where tumor growth was more than $25 \%$ or new lesions were found.

\section{Adverse reactions determinationevaluation}

Adverse reactions of chemotherapy were evaluated according to the World Health Organization adverse reaction degree standards for anti-cancer drugs. The drug dose was adjusted or drug administration was stopped when significant bone marrow inhibition or nontoxic effects appeared, according to the research plan. Adverse reaction for radiotherapy was defined according to the acute radiation reaction scoring criteria given by the Radiation Therapy Oncology Group. Acute radiation reactions such as those in the bone marrow, gastrointestinal tract, and urinary system were evaluated during or at the end of the treatment. Acute radiation injury was divided into 5 levels as follows: Level 0 , no reaction; Level 1 , the symptom is slight, treatment can be continued; Level 2, moderate symptoms that need drug therapy; Level 3, severe symptoms that need medication, and radiation therapy should be interrupted or stopped; Level 4, fatal injury, irreversible.

\section{Follow-up}

All patients had the first follow-up 1 to 2 months after the treatment. Within the first year, a follow-up was performed every 2 to 3 months. Between the first year and second year after the treatment, a follow-up was performed every 3 to 6 months. Thereafter, a follow- 
up was performed every 6 months. The overall response rate (ORR) was evaluated every 3 months. Follow-up included gynecology examination, chest radiography, abdominal and pelvic ultrasonography, CT or MRI, and cancer antigen 125 level measurements. The follow-up methods included outpatient recheck, telephone calls, and regular letters. The follow-up was performed until December 31, 2011.

\section{Statistical analysis}

The SPSS 15.0 statistical software package was used. Survival curve, progressionfree survival time, and overall survival time were calculated using the Kaplan-Meier method. Comparisons were conducted with the Log rank test. The $t$-test and chi-square test were used to compare response rate, survival rate, and adverse reactions between the two groups. The statistically significant difference was $\mathrm{P}<0.05$.

\section{RESULTS}

Table 1 shows the clinical data of the two groups. Twenty-two patients were in the chemoradiotherapy group, with ages ranging from 38 to 67 years, and a median age of 52 years. Twenty patients were in the chemotherapy group, with ages from 35 to 71 years, and a median of 50 years. The results show no statistical differences in age, clinical stage, histological grade, surgical history, and recurrence status between the two groups $(\mathrm{P}=0.936, \mathrm{P}=0$. $591, \mathrm{P}=0.815, \mathrm{P}=0.530, \mathrm{P}=0.499$, respectively).

\begin{tabular}{|c|c|c|c|}
\hline Clinical features & $\begin{array}{l}\text { Chemoradio-therapy } \\
\text { plus topotecan group }\end{array}$ & $\begin{array}{c}\text { Only } \\
\text { chemotherapy group }\end{array}$ & $P$ \\
\hline \multicolumn{4}{|l|}{ FIGO grade } \\
\hline II & 4 & 5 & \\
\hline III & 18 & 15 & 0.591 \\
\hline \multicolumn{4}{|l|}{ Histological grade } \\
\hline Poorly differentiated & 15 & 12 & \\
\hline Medium and highly differentiated & 7 & 8 & 0.815 \\
\hline \multicolumn{4}{|l|}{ Past surgery status } \\
\hline First operation & 14 & 12 & \\
\hline Second operation & 8 & 8 & 0.530 \\
\hline \multicolumn{4}{|l|}{ Recurrent imaging manifestation } \\
\hline Single pelvic mass & 12 & 9 & 0.499 \\
\hline Retroperitoneal lymph node metastasis & 4 & 5 & \\
\hline Both & 6 & 6 & \\
\hline
\end{tabular}

\section{Follow-up results}

The mean follow-up time was 18.5 months (2.0 to 37.7 months) in the chemoradiotherapy group and 10.8 months (1.5 to 29.6 months) in the chemotherapy group. Until June 2011, among the 19 patients in the chemoradiotherapy group who actually finished treatment, 4 patients were lost to follow-up. Among the 18 patients in the chemotherapy group who actually finished treatment, 3 patients were lost to follow-up. Follow-up rates were 79\% (15/19) and $83 \%(15 / 18)$, respectively. 


\section{Improved symptoms after treatment}

In the chemoradiotherapy group, 8 patients had vaginal bleeding. Symptoms improved after radiotherapy. Nineteen patients had pelvic, waist, and leg pain; the pain disappeared in 9 patients after radiation, whereas the pain was obviously alleviated in 6 patients. The effective rate of radiation when pain stopped was $79 \%(15 / 19)$. In the chemotherapy group, 5 patients had vaginal bleeding before chemotherapy, which disappeared completely in 2 patients after chemotherapy. Twelve patients had pelvic, waist, and leg pain. The pain disappeared in 3 patients after chemotherapy. The pain was obviously alleviated in 6 patients. The effective rate of chemotherapy when pain stopped was $75 \%(9 / 12)$. The two groups showed no significant difference $(\mathrm{P}>0.05)$.

\section{Curative effect}

Of the 22 patients in the chemoradiotherapy group (3DCRT and TPT combined therapy), 3 patients dropped out whereas 19 patients actually completed the treatment. Of the 20 patients in the chemotherapy group (single medicine TPT chemotherapy), 2 patients dropped out whereas 18 patients actually completed the treatment. Among the 19 patients of the chemoradiotherapy group who completed the treatment, 3 patients showed CR, 5 patients showed PR, and 5 patients showed SD. The total response rate was $42.1 \%(8 / 19)$, the CR rate was $15.8 \%(3 / 19)$, and the clinical benefit rate was $68.4 \%(13 / 19)$. Among the 18 patients of the chemoradiotherapy group who completed the treatment, 2 people showed PR and 2 showed SD. The total response rate was $11.1 \%(2 / 18)$, and the clinical benefit rate was $22.2 \%(4 / 18)$. In terms of total remission rate and clinical benefit rate, the two groups had significant difference $(\mathrm{P}=0.038, \mathrm{P}=0.038$, respectively (Table 2). The disease progression-free time was 9.8 months in the chemoradiotherapy group (range, 5.9 to 13.8) and 6.6 months in the chemotherapy group (range, 4.2 to 8.6). The two groups showed significant difference $(\mathrm{P}<0.001)$. The median survival time was 19.7 months for the chemoradiotherapy group and 12.5 months for the chemotherapy group. The two groups showed significant difference $(\mathrm{P}<0.05$; Figure 1$)$.

Table 2. Comparison of the curative effect of two groups of patients with platinum-resistant recurrent ovarian cancer.
\begin{tabular}{lcccr}
\hline Remission rate & $\begin{array}{c}\text { Chemoradiotherapy plus } \\
\text { topotecan group (N = 19) }\end{array}$ & $\begin{array}{c}\text { Only chemotherapy } \\
\text { group (N = 18) }\end{array}$ & $\chi^{2}$ & P \\
\hline Complete remission rate & $3(15.8)$ & 0 & 3.093 & 0.125 \\
Partial remission rate & $5(26.3)$ & $2(11.1)$ & 1.393 & 0.225 \\
Total remission rate & $8(42.1)$ & $2(11.1)$ & 4.502 & 0.038 \\
Clinical benefit rate & $13(68.4)$ & $4(22.2)$ & 7.943 & 0.008 \\
\hline
\end{tabular}

\section{Acute adverse reactions}

The acute adverse reactions in the chemoradiotherapy group mainly included gastrointestinal tract reaction, bone marrow suppression, and urinary systems reaction. The acute adverse reactions in the chemotherapy group mainly included bone marrow suppression and gastrointestinal reaction. The incidence of the acute adverse reaction showed no significant difference between both groups $(\mathrm{P}>0.05)$. With active symptomatic and support treatment, most patients can complete the treatment plan. There were no radiotherapy- and chemotherapy-related deaths (Table 3). 


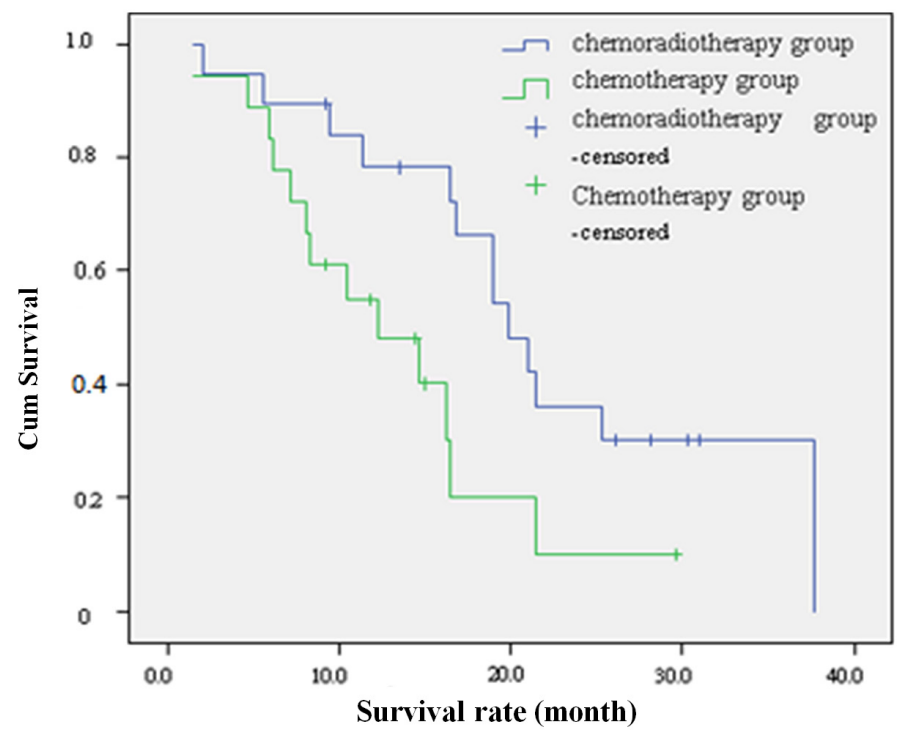

Figure 1. Survival curves of patients with platinum-resistant recurrent epithelial ovarian cancer and treated with chemoradiotherapy plus topotecan or topotecan alone.

Table 3. Comparison of toxicities between two groups of patients with platinum-resistant recurrent ovarian cancer.

\begin{tabular}{|c|c|c|c|c|c|c|c|c|c|c|}
\hline \multirow[t]{2}{*}{ Adverse reaction } & \multicolumn{5}{|c|}{ Chemoradiotherapy plus topotecan group } & \multicolumn{5}{|c|}{ Only chemotherapy group } \\
\hline & 0 & I & II & III & IV & 0 & I & II & III & IV \\
\hline Digestive tract reaction & 1 & 6 & 7 & 5 & 0 & 0 & 5 & 9 & 4 & 0 \\
\hline Hematology toxicity & 1 & 8 & 7 & 3 & 0 & 1 & 7 & 6 & 4 & 0 \\
\hline
\end{tabular}

\section{Around target radiation and chemotherapy organs at risk}

According to the OAR dosed volume graph, the OAR irradiation dose was obtained. The OAR irradiated doses were within the acceptable range. Therefore, 3DCRT can increase target exposure dose. At the same time, it can reduce the irradiation dose around target areas and protect the target organs (Table 4).

Table 4. Tolerance dose for organs at risk in the chemoradiotherapy plus topotecan group.

\begin{tabular}{lccc}
\hline Organ at risk & Median dose $(\mathrm{Gy})$ & \multicolumn{2}{c}{$\mathrm{TD}_{5 / 5}$ volume reference dose $(\mathrm{Gy})$} \\
\cline { 3 - 4 } & & $1 / 3$ & $3 / 3$ \\
\hline Liver & $12.9 \pm 7.7$ & 50 & 30 \\
Kidney & $15.6 \pm 4.3$ & 50 & 23 \\
Small intestine & $22.7 \pm 8.9$ & 50 & 40 \\
Spinal cord & $26.1 \pm 9.4$ & - & 65 \\
Bladder & $37.6 \pm 5.8$ & - & 60 \\
Rectum & $31.3 \pm 8.5$ & - & 52 \\
Femoral head & $22.4 \pm 4.8$ & 50 \\
\hline
\end{tabular}

$\mathrm{TD} 5 / 5$ refers to no more than $5 \%$ of the total patients experiencing severe radioactive damage caused by radioactive rays in 5 years after standard treatment. 


\section{DISCUSSION}

Epithelial ovarian cancer radiation therapy is a controversial topic. In the 1960s and 1970s, surgery plus full abdomen irradiation was the predominant treatment for ovarian epithelial carcinoma. It obtained certain therapeutic effects, but the incidence of adverse effects was also quite high (Dembo, 1992). Platinum-based chemotherapy achieved a good treatment effect. The adverse effects can be tolerated. Chemotherapy gradually replaced whole abdomen postoperative irradiation in ovarian epithelial carcinoma treatments. However, recurrent tumors, especially recurrent metastatic lesions in certain areas, such as retroperitoneal lymph node metastasis, pelvic metastasis, and recurrent lesions of vaginal residuals, have had poor sensitivity to chemotherapy. Radiation therapy can be used to effectively control residual or metastatic lesions, and it complements the effects of chemotherapy (Yang et al., 2012). With the recent clinical application of the 3DCRT technique, radiation therapy can further shrink the irradiation target compared with traditional routine radiotherapy. It can also increase the target dose and reduce the exposure dose of adjacent normal tissues and organs. It can increase the tumor control rates, and at the same time, greatly reduce the adverse reaction. It has given gynecological oncologists a new tool in the clinical treatment of epithelial ovarian carcinoma (Garsa et al., 2007; Sehouli et al., 2012)

TPT is a topoisomerase I inhibitor. It has no cross-resistance with paclitaxel and platinum, and has certain curative effects on recurrent patients with platinum resistance. Safra et al. (2007) evaluated the effect of $4 \mathrm{mg} / \mathrm{m}^{2} \mathrm{TPT}$ chemotherapy that was administered on days 1,8 , and 15 , every 28 days. TPT demonstrated efficacy and safety in recurrent ovarian cancer patients with platinum resistance. Results showed that the objective response rate was $23.8 \%$, the median disease progression-free time was 6.2 months, and the median survival time was 22.3 months. In the chemotherapy group of this study, 18 patients were treated with TPT chemotherapy, 2 patients showed PR, 2 patients showed SD, and the total benefit rate was $22.2 \%$ (4/18), all of which were consistent with the results by Safra et al. (2007). Therefore, TPT can serve as an effective treatment choice for platinum-resistant recurrent epithelial ovarian cancer.

Retroperitoneal lymph node metastasis is an important factor that affects the prognosis of patients with ovarian cancer. Lymph node excision can obviously prolong the disease progression-free time, but it cannot extend the overall survival time (Salet-Lizée and Alsary, 2008). Studies have found that platinum-based chemotherapy does not have the ideal curative effect on retroperitoneal lymph node metastases (Burghardt and Winter, 1989). Niu et al. (2000) discussed the use of intraperitoneal injection of low-dose cisplatin (short time) for the treatment of retroperitoneal lymph node metastasis of ovarian epithelial carcinoma lesions. Thirty patients were divided into two groups. The first group received intraperitoneal chemotherapy $48 \mathrm{~h}$ before operation; the second group received intraperitoneal chemotherapy 96 and $48 \mathrm{~h}$ before the operation. The spectrometric method was used for determining the concentration of cisplatin in the peritoneal and retroperitoneal lymph nodes, and pathological examination of resected lymph nodes was performed. Results showed that the peritoneal drug concentration was 4.14 and 2.50 times higher than the lymph node drug concentration in the two groups of patients, respectively. It can be seen that retroperitoneal lymph nodes have a poor response to chemotherapy compared with the peritoneum. Therefore, a more effective treatment for retroperitoneal lymph node metastasis is needed.

In 2010, the international union of obstetrics and gynecology performed a phase I clinical trial and reported the feasibility of a new treatment in patients with advanced ovar- 
ian cancer. At first, optimal debulking was performed; then, chemotherapy and full abdomen intensified radiation therapy were performed. Ten patients with advanced ovarian cancer were selected. With the completion of the first tumor reduction surgery, paclitaxel-platinum chemotherapy was administered postoperatively. The abdominal, pelvic, and lymphatic drainage areas of the abdominal aorta were treated with intensified radiation therapy. The single dose was $1.5 \mathrm{~Gy}$; the total dose was $30 \mathrm{~Gy}$.

The results showed that no patient dropped out because of adverse reactions after radiotherapy, and the median survival time was 23 months (Rochet et al., 2010). This suggests that full abdomen intensified radiation therapy can be used as an effective consolidating treatment of postoperative chemotherapy in advanced ovarian cancer patients. Bignardi et al. (2011) reported that 19 patients with recurrent ovarian cancer having retroperitoneal lymph node metastasis, who could not be operated on, received stereotactic radiotherapy. The prescribed dose was $45 \mathrm{~Gy}$. A good clinical control rate was achieved, and acute and chronic adverse reactions were mild.

Lee et al. (2011) found that radiation had a higher efficiency than chemotherapy for nodular metastatic lesions and isolated lesions of patients with recurrent ovarian cancer. These authors carried out a retrospective study that included 67 patients with recurrent ovarian cancer: 50 patients underwent radiotherapy and 17 patients underwent chemotherapy. The total effective rate was $64 \%$ in the radiation group and $16.7 \%$ in the chemotherapy group. The overall survival rate was 38 months in the radiotherapy group and 18 months in the chemotherapy group.

The median progression-free survival time was 6 months in the radiotherapy group and 5 months in the chemotherapy group. A significant difference was found in the total effective rates between the two groups. The median overall survival time and progression-free survival time showed no significant difference. In this study, the total effective rate and clinical benefit rate in the radiation and chemotherapy group were significantly higher than those in the only chemotherapy group. The two groups showed a significant difference $(\mathrm{P}=0.038, \mathrm{P}=$ 0.038), which was consistent with the research results by Lee et al. (2011). The results showed that 3DCRT combined with occasional TPT chemotherapy could further improve the curative effect in patients with recurrent ovarian cancer.

In summary, after tumor cell reduction surgery and postoperative chemotherapy in epithelial ovarian carcinoma patients, the pelvic recurrence or retroperitoneal lymph node metastatic lesions were resected difficultly, and the sensitivity to chemotherapy was poor. Conformal irradiation therapy can effectively increase the dose administered to local lesions in order to improve the local control rate and the long-term survival rate. Therefore, in the treatment of platinum-resistant recurrent epithelial ovarian cancer, 3DCRT combined with TPT chemotherapy can be used as an alternative therapy.

\section{REFERENCES}

Bignardi M, Navarria P, Mancosu P, Cozzi L, et al. (2011). Clinical outcome of hypofractionated stereotactic radiotherapy for abdominal lymph node metastases. Int. J. Radiat. Oncol. Biol. Phys. 81: 831-838.

Burghardt E and Winter R (1989). The effect of chemotherapy on lymph node metastases in ovarian cancer. Baillieres Clin. Obstet. Gynaecol. 3: 167-171.

Dembo AJ (1992). Epithelial ovarian cancer: The role of radiotherapy. Int. J. Radiat. Oncol. Biol. Phys. 22: 835-845.

Gardner GJ and Jewell EL (2011). Current and future directions of clinical trials for ovarian cancer. Cancer Control 18: 44-51.

Garsa AA, Andrade RS, Heron DE, Beriwal S, et al. (2007). Four-dimensional computed tomography-based respiratorygated whole-abdominal intensity-modulated radiation therapy for ovarian cancer: a feasibility study. Int. J. Gynecol. 
Cancer 15: 55-60.

Lee M, Kim SW, Lee SH, Paek J, et al. (2011). Comparison of the efficacy and toxicity between radiotherapy and chemotherapy in nodal and isolated nonnodal recurrence of ovarian cancer. Int. J. Gynecol. Cancer 21: 1032-1039.

Lorusso D, Pietragalla A, Mainenti S, Masciullo V, et al. (2010). Review role of topotecan in gynaecological cancers: current indications and perspectives. Crit. Rev. Oncol. Hematol. 74: 163-174.

Naumann RW and Coleman RL (2011). Management strategies for recurrent platinum resistant ovarian cancer. Drugs 71: 1397-1412.

Niu X, Peng Z, Ding S and Yang K (2000). The effect of intraperitoneal chemotherapy on retroperitoneal lymph node metastasis of ovarian cancer. Hua Xi Yi Ke Da Xue Xue Bao 31: 188-190.

Rochet N, Sterzing F, Jensen AD, Dinkel J, et al. (2010). Intensity-modulated whole abdominal radiotherapy after surgery and arboplatin/taxane chemotherapy for advanced ovarian cancer: phase I study. Int. J. Radiat. Oncol. Biol. Phys. 76: $1382-1389$

Safra T, Menczer J, Bernstein R, Shpigel S, et al. (2007). Efficacy and toxicity of weekly topotecan in recurrent epithelial ovarian and primary peritoneal cancer. Gynecol. Oncol. 105: 205-210.

Salet-Lizée D and Alsary S (2008). The place of lumo-aortic and pelvic lymph node dissection in the treatment of ovarian cancer. J. Chir. 4: 12S45-12S49.

Sehouli J and Oskay-Ozcelik G (2009). Current role and future aspects of topotecan in relapsed ovarian cancer. Curr. Med. Res. Opin. 25: 639-651.

Sehouli J, Runnebaum IB, Fotopoulou C, Blohmer U, et al. (2012). A randomized phase III adjuvant study in high-risk cervical cancer: simultaneous radiochemotherapy with cisplatin (S-RC) versus systemic paclitaxel and carboplatin followed by percutaneous radiation (PC-R): a NOGGO-AGO Intergroup Study. Ann. Oncol. 23: 2259-2264.

Yang B, Zhu L, Cheng H, Li Q, et al. (2012). Dosimetric comparison of intensity modulated radiotherapy and threedimensional conformal radiotherapy in patients with gynecologic malignancies: a systematic review and metaanalysis. Radiat. Oncol. 7: 197. 